\title{
Bayesian Estimation of Genotypes Means, Precision, and Genetic Gain Due to Selection from Routinely Used Barley Trials
}

\author{
Murari Singh,^ Adnan Al-Yassin, and Siraj Osman Omer
}

\begin{abstract}
Block designs are normally used in evaluation of crop varieties. The responses or yield data arising from designed trials in a crop variety improvement program are generally analyzed using linear mixed models under the frequentist paradigm. Such analysis ignores information on the genotypic parameters available from previous similar trials. Another approach with a relatively wider inferential framework is Bayesian, which integrates the prior information with the likelihood of current data. While the Bayesian approach has been implemented in numerous situations, stepwise presentation of its application in routine crop variety trials is not available. Illustrated with a dataset from a resolvable incomplete block design, this study provides a working tool for Bayesian analysis based on priors available from a series of crop variety trials. The posterior estimates of predicted values of mean of genotypes and precision, coefficient of variation, heritability, and genetic gain due to selection were obtained. The a posteriori mean of experimental error variance, coefficient of variation, and genotypic variance were lower for the Bayesian than the frequentist approach. The precision of a posteriori means was higher than that of predicted means under the frequentist approach. Accounting for incomplete blocks, rather than ignoring them, using a Bayesian approach showed a large reduction in estimates of error variance components and large increases in heritability and genetic gain. The current a posteriori distributions also serve as updated priors for future analysis. The stepby-step procedure presented here is recommended for routine analysis of variety trials.
\end{abstract}

International Center for Agricultural Research in the Dry Areas (ICARDA), P.O. Box 5466, Aleppo, Syria. Received 12 Feb. 2014. ^Corresponding author (m.singh@cgiar.org).

Abbreviations: DIC, deviance information criterion; GA, genetic advance; IBD, incomplete block design; REML, restricted maximum likelihood; RCBD, randomized complete block design; SDC, standard deviation components.

$I^{\mathrm{N}}$ N CROP VARIETY TRIALS and other experiments, suitable experimental designs are chosen to segregate the observed variability in the trait of interest, such as grain yield, into those due to systematic patterns in the experimental units, varieties or treatments, and (uncontrollable) experimental errors. The basics of experimental design and analysis of data are detailed in several standard texts (Cochran and Cox, 1957; Kempthorne, 1983; Fisher, 1990; Hinkelmann and Kempthorne, 2005). A number of standard statistical software packages such as Genstat (Payne, 2013), SAS (SAS Institute, 2011), and CycDesigN (Whitaker et al., 2009) and websites such as Design Resource Server (Parsad and Gupta, 2007) can generate the randomized plans for various experimental designs and carry out statistical analysis using linear models under the frequentist approach. Variety trials are generally conducted in complete or incomplete block designs such as an $\alpha$ design (Patterson and Williams, 1976; John and Williams, 1995). Experimental designs for elimination of heterogeneity in two dimensions are also frequently used, such as resolvable rowcolumn designs known as lattice squares and lattice rectangles (Federer, 1955; Cochran and Cox, 1957; Hinkelmann and Kempthorne, 2005). The $p$-rep designs with variable replications on

Published in Crop Sci. 55:501-513 (2015).

doi: 10.2135/cropsci2014.02.0111

Freely available online through the author-supported open-access option.

(C) Crop Science Society of America | 5585 Guilford Rd., Madison, WI 53711 USA

All rights reserved. No part of this periodical may be reproduced or transmitted in any form or by any means, electronic or mechanical, including photocopying, recording, or any information storage and retrieval system, without permission in writing from the publisher. Permission for printing and for reprinting the material contained herein has been obtained by the publisher. 
rectangular layouts were developed for spatially correlated errors (Cullis et al., 2006) and can be generated using the DiGGer package (Coombes, 2009). Augmented p-rep designs based on one-way incomplete blocks and suitable for multienvironment trials (Williams et al., 2011) can be generated by the CycDesigN software (Whitaker et al., 2009). Because the crop improvement process is long, varieties are evaluated in a series of trials over multiple locations and years, where in a current year only some varieties from previous years are retained and some new varieties are included in the trials. Considered over the multiple environments, suitably chosen mixed models are used to analyze the resulting unbalanced datasets. Smith et al. (2005) gives a detailed description on handling such trials using a frequentist approach.

In well-established crop improvement programs, information on genotypic and environmental variability is available from the statistical analysis of routinely conducted variety trials. The frequentist paradigm does not focus on using such prior information gathered from previous or other trials on similar genotypic material. However, the Bayesian paradigm is oriented to use such prior information to further empower the statistical inference that could be drawn from the current trial. For instance, data on the same genotypes evaluated on earlier occasions and or along with other genotypes drawn from a germplasm pool or population can be used. Commonly used prior information, in terms of statistical distribution of the various parameters associated with experimental design and genetic material, can be obtained using a Bayesian method. The parameters of a priori distributions may be known or may themselves follow distributions with known parameters and so on along a hierarchy of parameters. The series of already-conducted trials may provide information on mean and variance components of the random factors involved in an experimental design to evaluate the set of genotypes. In this context, the distributions of estimates of variance components of various effects available from a set of 20 trials provided a basis for Bayesian analysis. Although the Bayesian approach makes use of prior information, in the form of statistical distribution of the parameters while the frequentist approach does not, a question arises as to what degree of difference can be seen. The answer depends on the choice of priors and the dataset. Illustrative examples (Lindley, 1971; Gelman et al., 2004 [page 47]) show that the Bayesian estimate or posterior expectation of the parameter of interest is derived as a weighted estimate that lies between the frequentist estimate and the prior and thus, shows the usefulness of the Bayesian approach (see also Leonard, 1975; Edwards and Jannink, 2006).

The Bayesian approach is well described in the literature and standard texts (Gelman et al., 2004; Carlin and Louis, 2009). In short, it is an integration of prior information with current data to obtain posterior information in terms of statistical distribution and can be described as follows. Let a single parameter $\theta$ be inferred using an observed data vector $\underline{\mathbf{y}}=\left(\gamma_{1}, \ldots, \gamma_{n}\right)^{\prime}$, and probability distribution or the likelihood of observing $\mathbf{y}$ based on a value of $\theta$ be denoted by $f(\mathbf{y} \mid \theta)$ as a function of $\theta$. The prior information on $\theta$ that might result from a series of already-observed datasets serves as a degree of belief in $\theta$ and is described in terms of its probability distribution function, for example $g(\theta)$, called an a priori distribution of $\theta$ or simply a prior for $\theta$. The inference on $\theta$ is obtained in terms of the probability distribution of $\theta$ given the data $\mathbf{y}$ and is expressed as $f(\theta \mid \gamma) \propto g(\theta) f(\gamma \mid \theta)$, called the a posteriori or simply a posterior density function of $\theta$, obtainable from the famous Bayes' Theorem available in standard texts (Gelman et al., 2004). Thus, the a posteriori distribution is an integration of prior information with likelihood of the current data. This integration distinguishes the Bayesian from the frequentist approach. This a posteriori density is used to obtain the conditional expected value of $\theta$ as an estimate of $\theta$, standard error, and its confidence intervals. Powerful computing algorithms easily extend this approach to more than one parameter.

Furthermore, there might be more than one source of prior information in a breeding program, including classes of priors recommended for variance or standard deviation components. A question naturally arises as to which is the best prior among the set of priors considered and a suitable criterion is required for the selection. As the crop improvement process is normally long, prior information for a future investigation could be enhanced by the current posterior distribution and thus, in an ongoing breeding program, the Bayesian priors would continue going through regular and possibly more informative updates.

Theobald et al. (2002) implemented a Bayesian approach to predict regional and local-area yields from crop variety trials. Theobald and Talbot (2002) introduced a full Bayesian formulation for choosing the crop variety and fertilizer level using the enhanced knowledge of the crop and soil conditions. Recent trends in quantitative genetic data analysis have been to incorporate the Bayesian paradigm. Based on local factors in the fieldadjusted means of wheat (Triticum aestivum L.) genotypes from an experimental design, de los Campos et al. (2009) and Crossa et al. (2010) applied a Bayesian approach involving independent priors for linear effects and associated variance components parameters to predict the genetic value using pedigree and molecular marker information in a high dimension. Edwards and Jannink (2006) applied the Bayesian approach to model heterogeneity of error variances and genotype $\times$ environment interaction variances, since, in a frequentist approach for exploring multienvironment trials data, the true variances would be generally unknown and replacing them by their estimates 
introduces errors of estimation in the parameters. The logarithms of interaction variances were first expressed as an additive model of random effects of the classifications, genotype, and environment, and the variances of these effects were assigned suitable priors. For data from small complete block designs, Forkman and Piepho (2012) found that Bayesian analysis resulted in the smallest root meansquare error and more accurate coverage of prediction intervals for means compared with best linear unbiased estimators and empirical best linear unbiased predictors (BLUPs). For relatively more complicated situations, using decomposition of a multivariate normal distribution in terms of univariate conditional distributions, Hallander et al. (2010) developed a Bayesian tool and estimated genetic parameters of complex pedigrees of Scots pine (Pinus sylvestris $\mathrm{L}$.) with additive and dominance polygenic relationships and simulated pedigree with genomic relationships calculated from a dense marker map. Mathew et al. (2012) proposed a fast adaptive Markov chain Monte Carlo sampling algorithm for the estimation of genetic parameters using a linear mixed model with several random effects and illustrated with simulated data.

The major points in genotypic evaluation are the estimation of predicted values of genotypic mean, broadsense heritability on a mean basis, and genetic advance (GA) or gain due to selection. The expression of heritability for randomized complete block designs (RCBDs) was adopted for incomplete block designs (IBDs) by Singh et al. $(2012,2013)$ in terms of genotypic variance, ploterror variance, and number of replications. The bias of such an estimator on a plot basis was studied by Singh and Ceccarelli (1995). Piepho and Möhring (2007) noted that the heritability expression of Singh et al. (2013) was overestimated for IBDs and suggested using an alternative expression given by Cullis et al. (2006) in terms of genotypic variance and average pair-wise variance of BLUPs. However, the properties of such an estimator have not been studied. Genetic advance is obtained by the same expression in terms of heritability and selection intensity. Piepho and Möhring (2007) also studied the realized gain through simulation. Since the distribution of parameters of interest is obtained in terms of simulated values of the parameters, these expressions are included in the Bayesian model section and the $\mathrm{R}$-codes section of the appendix (A.7 and A.9, respectively).

While there are many applications of Bayesian approaches in crop improvement trials, we believe there are limited worked examples available for the Bayesian version of data analysis from routinely implemented block designs. The objectives of this study is to systematically present, along with an extraction of prior information based on an observed series of crop variety trials other than the current trial, the methodology of analyzing data from experiments designed in RCBDs and one-way IBDs, including selection of the best priors from chosen candidate priors. It also aims to provide a working example for Bayesian analysis of data from an IBD and a RCBD to estimate the experimental error variance, coefficient of variation, predicted means of genotypes (with standard errors and confidence intervals), broad-sense heritability on mean basis, genetic gain due to selection, realized gain due to selection, and ranking of genotypes for selection. The necessary codes of the WinBUGS (Spiegelhalter et al., 2003) and R-package (R Development Core Team, 2009) are provided.

\section{MATERIALS AND METHODS}

We used the following dataset on grain yield.

\section{Dataset (Incomplete Block Designs)}

A set of 30 barley (Hordeum vulgare L.) genotypes, including two common checks, were evaluated in an $\alpha$ design in six blocks of size five each and with three replications. The experiment was planted on 1 November 2011 at Tel Hadya, northern Syria. Seeds were sown to germinate approximately 250 plants per $\mathrm{m}^{2}$ in eight-row plots of 7.5-m length and interrow distance of 0.2 $\mathrm{m}$. Only the six central rows were harvested, and data on grain yield was recorded in $\mathrm{kg} \mathrm{ha}^{-1}$.

\section{Data for A Priori Information}

A series of 20 trials with similar experimental designs was available, which provided the prior information. These datasets were analyzed using the restricted maximum likelihood (REML) method, a frequentist approach. The dataset on trial means, estimates of variance components for replication, blocks within replications, genotype, and experimental errors were obtained. Since we also wanted to illustrate the example for RCBD, the same 20 sets were reanalyzed by ignoring the incomplete blocks and estimates of variance components were tabulated.

\section{Data Analysis A Priori Distributions}

With the availability of 20 sets of values on trial mean and variance components, statistical distributions were fitted to them. For the square root of the variance components, that is, standard deviation components (SDC), a normal distribution was fitted in Genstat software (Payne, 2013). The goodness-of-fit statistics derived as chi-square statistics were obtained from observed frequencies and normal distribution-based fitted frequencies available in the DISTRIBUTION directive of Genstat software and $p$-values for deviance as shown in Table 1 . The distribution of each SDC was normal $(p>0.01)$ except for the SDC for replication effects in IBD data. These resulted in deciding on the a priori distributions. Since SDCs are positive, we assigned the positive part of the normal distributions as the a priori distribution for all SDCs with respective variances or precision estimated from the IBD data and for RCB data obtained by ignoring incomplete blocks. Further, since a priori information may arise from several sources, and one may be interested in selecting the most suited prior, we also included a number of priors explored or recommended in Gelman (2006). A number of such priors evaluated in this study are also included in Table 2. 
Table 1. Fitting of normal distribution to means and standard deviation (SD) components of genotype, blocks within replications, replication, and experimental error estimated from a series of 20 winter barley trials using a frequentist approach.

(a) Incomplete block design ${ }^{\dagger}$

\begin{tabular}{llcccc}
\hline & $\begin{array}{c}\text { Trial } \\
\text { mean } \mu\end{array}$ & $\begin{array}{c}\text { Replica- } \\
\text { tion } \sigma_{\mathrm{r}}\end{array}$ & $\begin{array}{c}\text { Geno- } \\
\text { typic } \\
\text { SD } \sigma_{\mathrm{g}}\end{array}$ & $\begin{array}{c}\text { Experi- } \\
\text { mental } \\
\text { error } S \sigma_{\mathrm{e}}\end{array}$ & $\begin{array}{c}\text { Incomplete } \\
\text { blocks } \\
\text { within repli- } \\
\text { cations } \sigma_{\mathrm{b}}\end{array}$ \\
\hline Mean & 2.26 & 0.1812 & 0.1205 & 0.4061 & 0.4037 \\
SD & 0.3585 & 0.2199 & 0.1152 & 0.0729 & 0.1914 \\
Chi-square $^{\ddagger}$ & 2.05 & 42.78 & 4.72 & 1.32 & 3.04 \\
-value $^{\S}$ & 0.152 & $<0.001$ & 0.030 & 0.251 & 0.081 \\
\hline
\end{tabular}

(b) Complete block designs (ignoring incomplete blocks in the trials used in case a) ${ }^{\dagger}$

\begin{tabular}{llccl}
\hline & $\begin{array}{c}\text { Trial } \\
\text { mean } \mu\end{array}$ & $\begin{array}{c}\text { Replica- } \\
\text { tion } \sigma_{\mathrm{r}}\end{array}$ & $\begin{array}{c}\text { Experi- } \\
\text { typic SD } \\
\sigma_{\mathrm{g}}\end{array}$ & $\begin{array}{c}\text { mental } \\
\text { error SD } \\
\sigma_{\mathrm{e}}\end{array}$ \\
\hline Mean & 2.26 & 0.1431 & 0.1455 & 0.5605 \\
SD & 0.3585 & 0.1266 & 0.1331 & 0.1533 \\
Chi-square $^{\ddagger}$ & 2.05 & 0.69 & 1.06 & 4.57 \\
-value $^{\S}$ & 0.152 & 0.406 & 0.303 & 0.033 \\
\hline
\end{tabular}

${ }^{\dagger} \mu=$ trial mean; $\sigma_{r}=$ standard deviation component for replication effects; $\sigma_{g}=$ standard deviation component for genotype effects; $\sigma_{e}=$ standard deviation component for experimental errors; $\sigma_{b}=$ standard deviation component for incomplete blocks within replications effects.

‡ Chi-square with one degree of freedom.

$\S p$-value for normality.

In the following, we describe the main steps of the Bayesian analysis for data from an IBD. The related statistical models and choice of parameters, Win2BUGS, and R-language codes are detailed in the appendix.

Step 1. Identify the model for analysis of data. For IBD data, a linear model was described in terms of general mean, effects of replications, effects of incomplete blocks within replications, genotype effects, and experimental errors. These effects and errors were assumed to be random variables and thus had variance or standard deviation components.

Step 2. Identify the parameters of interest. The parameters studied were two expressions of heritability, three parameters measuring GA, or gain at 20\% selection intensity (Singh et al., 2013, Cullis et al., 2006), and realized gain as per Piepho and Möhring (2007).

Step 3. Begin with a prior set given for the mean and standard distributions, for example from Table 1.

Step 4. Prepare a text file, with extension 'bug,' containing the codes for data model, priors, and parameters to be evaluated (Steps 1-3). These are shown in Appendix, A.7.

Step 5. Prepare another file with R-codes including (i) access to the required libraries for running the Win2BUGS model file, (ii) reading the vectors on replications, incomplete blocks, genotypes and response vectors from IBD data file, (iii) set the initial values of random numbers generated, (iv) set up parameters, and (v) set up 'bugs()' function using data, initial values, parameters, the model file 'IBD.bug,' number of chains, number of iterations, and simulations in the R-package. These lines are shown in Appendix A.9.

Step 6. Run Steps 4 and 5 for each set of priors (see Appendix A.8) and note the deviance information criterion (DIC) values for selecting the best prior. Select the prior set with the lowest DIC value.

Step 7: For the prior set selected, modify the 'bug' extension file (Step 4) and again run Step 5 to save the simulated values of various parameters (e.g., predicted means, heritability, and GA) and to carry out calculations on ranks and heritability using simulated values (Cullis et al., 2006). The R-package (boot) with 1000 bootstrap replications was used to generate standard errors of heritability and GA using the method of Cullis et al. (2006).

For RCBDs, the effects and variance components for incomplete blocks can be ignored in the above steps.

\section{RESULTS Selection of Priors}

The frequentist (non-Bayesian) approach was followed using the REML method for analysis of the IBD dataset. By ignoring the effects of incomplete blocks within replications, the results for RCBD were obtained. The various statistics computed are presented along with the statistics using the Bayesian approach.

The choice of priors for Bayesian analysis was made from the statistics for the IBD and RCBD models (Table 2). For the IBD, of the six prior sets, $\mathrm{P}_{0}$ through $\mathrm{P}_{5}$, the DIC value was smallest for $\mathrm{P}_{0}$ (49.68), the prior set based on the means, and variances from data of the 20 trials of winter barley. The prior set derived from past data was more suitable to assign belief for the parameters than the priors based on large variances (Gelman, 2006). When the incomplete blocks were ignored (i.e., the RCBD data was modeled), the prior set based on previously collected data also gave the smallest DIC value (99.05), although in this case, DIC values for the other prior sets were very close. Therefore, the prior set $\mathrm{P}_{0}$ for IBD and $\mathrm{Q}_{0}$ for $\mathrm{RCB}$ were used to predict performance of genotypes and to estimate genetic parameters.

\section{Error Variability Using Selected Priors}

For the prior set $\mathrm{P}_{0}$ for the IBD model accounting for the effects of the incomplete blocks and $\mathrm{Q}_{0}$ for the $\mathrm{RCB}$ model obtained by ignoring the incomplete blocks from IBD model. Table 3 gives estimates of error variances, trial means, and coefficients of variation measuring the field heterogeneity under the Bayesian and frequentist approaches. Table 3 also gives estimates of genotypic variance and heritability on mean basis using expressions $h^{2}$ or $h_{\mathrm{C}}^{2}$, GA due to selection of the top $20 \%$ of genotypes using each of the two heritability estimates, as well as based on simulations. For the IBD data, the Bayesian estimate of experimental error variance, that is, a posteriori expected value of the 
Table 2. Priors chosen for mean and standard deviation component and discrepancy statistics for selection of the priors for IBD and RCB datasets.

\begin{tabular}{|c|c|c|c|c|c|c|c|c|c|}
\hline \multirow{2}{*}{$\begin{array}{l}\text { Prior } \\
\text { sets }\end{array}$} & \multicolumn{5}{|c|}{ Parameters ${ }^{\dagger}$} & \multicolumn{4}{|c|}{ Discrepancy statistics ${ }^{\ddagger}$} \\
\hline & $\mu$ & $\sigma_{\mathrm{r}}$ & $\sigma_{\mathrm{g}}$ & $\sigma_{e}$ & $\sigma_{\mathrm{b}}$ & $\bar{D}$ & $\hat{D}$ & $p_{\mathrm{D}}$ & DIC \\
\hline \multicolumn{10}{|c|}{ (a) Priors used for parameters of IBD } \\
\hline$P_{0}$ & $\mathrm{~N}(2.26,7.78)^{+}$ & $\mathrm{N}(0,20.67)^{+}$ & $\mathrm{N}(0,75.35)^{+}$ & $\mathrm{N}(0,188.17)^{+}$ & $\mathrm{N}(0,27.29)^{+}$ & 15.39 & -18.90 & 34.29 & 49.68 \\
\hline$P_{1}$ & $\mathrm{~N}(0, .00001)$ & Uniform(0, 10) & Uniform $(0,10)$ & Uniform $(0,10)$ & Uniform $(0,10)$ & 25.49 & -7.28 & 32.77 & 58.27 \\
\hline $\mathrm{P}_{2}$ & $\mathrm{~N}(0, .00001)$ & $\mathrm{N}(0,0.01)^{+}$ & $\mathrm{N}(0,0.01)^{+}$ & $N(0,0.01)^{+}$ & $\mathrm{N}(0,0.005)^{+}$ & 25.60 & -7.24 & 32.84 & 58.44 \\
\hline $\mathrm{P}_{3}$ & $\mathrm{~N}(0, .00001)$ & $t(0,5,2)^{+}$ & $t(0,5,2)^{+}$ & $t(0,5,2)^{+}$ & $t(0,5,2)^{+}$ & 26.25 & -6.17 & 32.42 & 58.67 \\
\hline $\mathrm{P}_{4}$ & $\mathrm{~N}(0, .00001)$ & $t(0,2,4)^{+}$ & $t(0,2,4)^{+}$ & $t(0,2,4)^{+}$ & $t(0,2,4)^{+}$ & 25.90 & -6.82 & 32.72 & 58.62 \\
\hline $\mathrm{P}_{5}$ & $\mathrm{~N}(0, .00001)$ & Gamma(.5, .5) & Gamma $(.5, .5)$ & Gamma(.5, .5) & Gamma(.5. 5) & 22.25 & -17.43 & 39.67 & 61.92 \\
\hline
\end{tabular}

(b) Priors used for parameters of RCB (ignoring blocks)

\begin{tabular}{|c|c|c|c|c|c|c|c|c|}
\hline Q0 & $\mathrm{N}(2.26,7.78)^{+}$ & $\mathrm{N}(0,62.4)^{+}$ & $\mathrm{N}(0,56.4)^{+}$ & $\mathrm{N}(0,42.6)^{+}$ & 86.25 & 73.44 & 12.81 & 99.05 \\
\hline Q1 & $\mathrm{N}(0, .00001)$ & Uniform $(0,10)$ & Uniform $(0,10)$ & Uniform $(0,10)$ & 85.32 & 70.81 & 14.50 & 99.82 \\
\hline Q2 & $\mathrm{N}(0, .00001)$ & $\mathrm{N}(0,0.005)^{+}$ & $\mathrm{N}(0,0.01)^{+}$ & $N(0,0.01)^{+}$ & 85.14 & 70.69 & 14.45 & 99.59 \\
\hline Q3 & $\mathrm{N}(0, .00001)$ & $t(0,5,2)^{+}$ & $t(0,5,2)^{+}$ & $t(0,5,2)^{+}$ & 85.18 & 70.93 & 14.25 & 99.43 \\
\hline Q4 & $\mathrm{N}(0, .00001)$ & $t(0,2,4)^{+}$ & $t(0,2,4)^{+}$ & $t(0,2,4)^{+}$ & 85.07 & 70.50 & 14.57 & 99.63 \\
\hline Q5 & $\mathrm{N}(0, .00001)$ & Gamma(.5, .5) & Gamma(.5, .5) & Gamma(.5, .5) & 76.00 & 51.66 & 24.35 & 100.35 \\
\hline
\end{tabular}



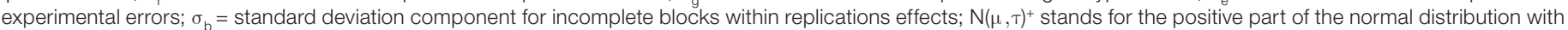

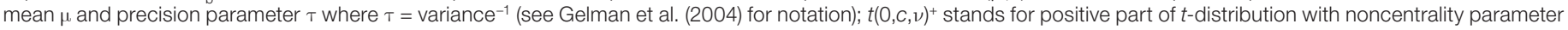
$c$ and the degree of freedom $\nu$.

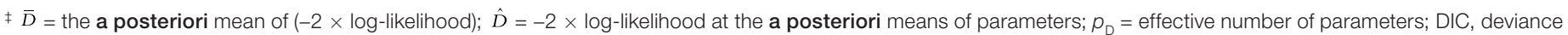
information criterion, smallest DIC values shown in bold.

Table 3. A posteriori means, credible intervals (CI), estimates of variance components, heritability, and genetic advance due to selection.

\begin{tabular}{|c|c|c|c|c|c|c|c|c|}
\hline \multirow[b]{2}{*}{$\begin{array}{l}\text { Incomplete } \\
\text { blocks effects }\end{array}$} & \multirow[b]{2}{*}{ Parameters $^{\dagger}$} & \multicolumn{5}{|c|}{ Bayesian approach } & \multicolumn{2}{|c|}{ Frequentist approach } \\
\hline & & $\begin{array}{l}\text { Posterior } \\
\text { mean }\end{array}$ & $\begin{array}{l}\text { Standard } \\
\text { error }\end{array}$ & Median & $\begin{array}{c}95 \% \mathrm{Cl}^{\ddagger} \\
\text { lower }\end{array}$ & $\begin{array}{c}95 \% \mathrm{Cl}^{\ddagger} \\
\text { upper }\end{array}$ & Estimate & $\begin{array}{l}\text { Standard } \\
\text { error }\end{array}$ \\
\hline \multirow{9}{*}{$\begin{array}{l}\text { Accounted } \\
\text { (incomplete } \\
\text { block design) }\end{array}$} & Error variance, $\sigma_{e}^{2}$ & 0.0628 & 0.0125 & 0.0614 & 0.0428 & 0.0914 & 0.0704 & 0.0153 \\
\hline & Trial mean, $\mu$ & 1.98 & 0.026 & 1.98 & 1.93 & 2.04 & 1.98 & \\
\hline & $\mathrm{CV} \%{ }^{\S}$ & 12.6 & 1.23 & 12.5 & 10.4 & 15.2 & 13.4 & \\
\hline & Genotypic variance, $\sigma_{\mathrm{g}}^{2}$ & 0.0404 & 0.0166 & 0.0387 & 0.0127 & 0.0782 & 0.0463 & 0.0203 \\
\hline & Heritability, $h^{2}$ & 0.635 & 0.1275 & 0.657 & 0.323 & 0.817 & 0.664 & 0.1215 \\
\hline & Heritability, $h_{\mathrm{C}}^{2}$ & 0.627 & 0.0018 & 0.627 & 0.623 & 0.630 & 0.620 & \\
\hline & $\mathrm{GA}(0.2) \%\left(h^{2}\right)$ & 11.2 & 3.32 & 11.3 & 4.6 & 17.8 & 12.4 & \\
\hline & $\mathrm{GA}(0.2) \%\left(h_{\mathrm{C}}^{2}\right)$ & 11.7 & 0.032 & 11.7 & 11.6 & 11.8 & 12.0 & \\
\hline & GA(0.2)\% (Simulation) & 13.9 & 2.75 & 14.0 & 8.1 & 19.0 & & \\
\hline \multirow{9}{*}{$\begin{array}{l}\text { Ignored } \\
\text { (randomized } \\
\text { complete } \\
\text { block design) }\end{array}$} & $\sigma_{e}^{2}$ & 0.1459 & 0.0265 & 0.1432 & 0.1014 & 0.2042 & 0.1380 & 0.0256 \\
\hline & $\mu$ & 1.99 & 0.040 & 1.99 & 1.91 & 2.07 & 1.98 & \\
\hline & CV\% & 19.2 & 1.73 & 19.1 & 16.1 & 22.8 & 18.7 & \\
\hline & $\sigma_{\mathrm{g}}^{2}$ & 0.0216 & 0.0189 & 0.0179 & 0.0001 & 0.0668 & 0.0373 & 0.0235 \\
\hline & $h^{2}$ & 0.274 & 0.1907 & 0.272 & 0.001 & 0.626 & 0.449 & 0.1774 \\
\hline & $h_{\mathrm{C}}^{2}$ & 0.281 & 0.0048 & 0.280 & 0.271 & 0.290 & 0.449 & \\
\hline & $\mathrm{GA}(0.2) \%\left(h^{2}\right)$ & 5.4 & 4.18 & 4.9 & 0.0 & 14.4 & 9.1 & \\
\hline & $\mathrm{GA}(0.2) \%\left(h_{\mathrm{C}}^{2}\right)$ & 5.6 & 0.061 & 5.6 & 5.5 & 5.7 & 9.1 & \\
\hline & GA(0.2)\% (Simulation) & 9.1 & 4.68 & 9.4 & 0.6 & 17.5 & & \\
\hline
\end{tabular}

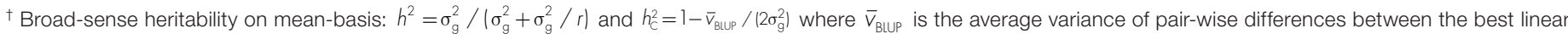

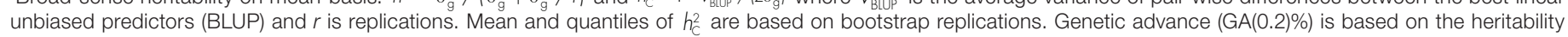
shown in parenthesis.

$\ddagger \mathrm{Cl}$, credible interval.

$\S \mathrm{CV}$, coefficient of variation.

error variance given the plot-wise response data under the assumed linear model and a priori distributions set $\mathrm{P}_{0}$, was lower $(0.0628 \pm 0.0125)$ than the frequentist error variance estimate $(0.0704 \pm 0.0153)$. The Monte Carlo errors in all parameters of Table 3 (and also other tables to follow) were very small, indicating reliable numerical approximation based on 1 million iterations. The genotypic variance, heritability estimates and GA estimates of the Bayesian approach were lower than for the frequentist approach. Since the Bayesian concept allows inferring soundly in 
terms of probability statements on the parameters of interest, their 95\% Bayesian confidence intervals, called credible intervals, are also given. The two a posteriori estimates of broad-sense heritability on mean basis, $h^{2}$ and $h_{\mathrm{C}}^{2}$, were very close to each other ( 0.64 vs. 0.63 , respectively), while in the frequentist approach, $h^{2}$ was slightly higher than $h_{\mathrm{C}}^{2}$ (0.66 vs. 0.62 , respectively). The GAs based on $h^{2}$ and $h_{\mathrm{C}}^{2}$ were close (11.2 vs. $11.7 \%$, respectively), but the simulated value was considerably higher (14\%).

One advantage of a resolvable design - where a set of incomplete blocks can be physically grouped to form a full replication, for example a lattice design or an $\alpha$ design - is that one can assess role of the incomplete blocks by analyzing the data after ignoring the incomplete blocks. There was a considerable reduction in error variance $\left(\sigma_{\mathrm{e}}^{2}\right)$ and increase in genetic variance $\left(\sigma_{\mathrm{g}}^{2}\right)$ when the incomplete blocks were accounted for (i.e., the IBD model) compared to when they were ignored (i.e., the RCBD model). As a result, there was a substantial increase in estimates of heritability of grain yield ( 27 or 28 to $64 \%$ for the two expressions, respectively) and the GA (5.4-9.1 to $13.9 \%$ for the three estimates of GA), reflecting the effectiveness of conducting experiments with incomplete blocks (Table 3).

\section{Predicted Values and Ranks of the Entries}

For the IBD dataset, the a posteriori means of the genotypes, 95\% credible intervals, and their a posteriori ranks from alternative methods are presented in Table 4, along with the predicted means for the frequentist approach as well as the RCB model. The precision of the means was higher for the Bayesian than the frequentist approach (average standard error of 0.128 vs. 0.160 for IBD and 0.130 vs. 0.168 for RCBD). Correlation between the predicted values under the two approaches was $0.994(p<0.001)$ for the IBD model, while the maximum difference in the predicted means was up to $4.6 \%$. Correlation between the means of the Bayesian approach for IBD and RCBD data was $0.817(p<0.001)$, reflecting the substantial difference in predicted values by accounting for the incomplete block differences. The performance of the genotypes for the Bayesian approach was also given in terms of percentiles of the predicted values and of their ranks. This was possible due to availability of a large number of simulated values under the Bayesian model (see Appendix A-9 for the R-codes). Denoting the genotypes numbered 1 through 30 by G1 through G30, the top six genotypes were G14, G19, G18, G5, G20, and G2 (G2 and G20 had equal means) using the Bayesian approach on IBD, which were also selected under the frequentist approach. Selection based on quantiles of ranks of the genotypes appeared quite desirable, instead of using only means, which is possible due to the availability of distribution of ranks from the computation process. When selecting the genotypes based on distribution of ranks, the top six genotypes using the a posteriori mean ranks or median of the ranks were also G14, G19, G18, G5, G2, and G20. While the selection of genotypes would be based on a posteriori mean rank or mean value, the distribution of ranks at the extreme quantiles could also be useful. Those genotypes that did not reach a high rank (toward one), near 2.5 or $5 \%$ percentiles, such as G10, G22, G29, or even G30, may not be selected. The ranks at percentiles 95 or $97.5 \%$ show how poor a good genotype, selected on average, could be-for example, G14's rank could go down to eight (95\% percentile) or 11 (97.5\% percentile), and similarly, G19 could, at worst, be ranked 12 (95\% percentile) or 15 (97.5\% percentile). Such a rank perspective assessment of genotypes is not possible in the frequentist approach as distributions of genotypes ranks are not applicable.

\section{Updating the Priors}

Current trial estimates of variance components can be used along with prior trials to develop prior distributions of the SDCs for similar future trials; however, the a posteriori distributions of the parameters naturally provide an update of the a priori distributions in the light of current datasets. The a posteriori means and precision $\left(\right.$ variance $^{-1}$ ) of SDCs (Table 5) compare with their a priori versions (Table 2). The precision parameters of the SDCs were considerably increased in the a posteriori distributions for the IBD as well as for RCBD datasets. This update, when implemented as a priori information for analysis of data collected in future trials on winter barley, may be expected to provide an even more realistic assessment of the barley genotypes. While the a posteriori distribution variance for the trial mean $\mu$ will also be available from the Bayesian computation, such an estimate would not be an appropriate prior for variance of mean $\mu$ for a new trial, which, in reality, would have no possibility of being implemented in exactly the same location and year. However, a reasonable prior variance on the mean of a new trial (e.g., the 22nd trial) would be the variance among means of the trials available so far (e.g., the first 21 trials in this case).

\section{DISCUSSION}

Germplasm improvement programs routinely conduct field experiments to estimate the performance of fixed genotypes or varieties and compare with a control or local check genotypes (comparative viewpoint) or to estimate parameters of the population such as heritability using a frequentist approach with a comparative or exploratory view (exploratory viewpoints). The data generations under these two views are based on assumed fixed effects for the genotypes and random effects, respectively (Hinkelmann, 1975). Crop breeders and geneticists keep changing the genotype base in their evaluation over time by including new or promising genotypes in evaluation and by discarding the poorly performing ones. The interest lies in prediction 
Table 4. Predicted values of the genotypes means and their ranks for a Bayesian approach and means for a frequentist approach for the dataset using incomplete block design (IBD) and randomized complete block design (RCBD) models.

\begin{tabular}{|c|c|c|c|c|c|c|c|c|c|c|c|c|c|}
\hline \multirow{3}{*}{$\begin{array}{l}\text { Rank of a } \\
\text { posteriori } \\
\text { mean }\end{array}$} & \multirow{3}{*}{$\begin{array}{l}\text { Geno- } \\
\text { type }\end{array}$} & \multicolumn{9}{|c|}{ 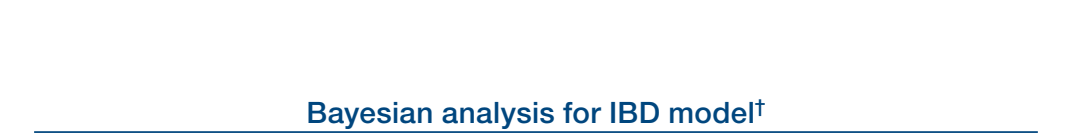 } & \multirow{3}{*}{ 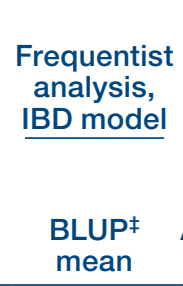 } & \multirow{3}{*}{$\begin{array}{c}\begin{array}{c}\text { Bayesian } \\
\text { analysis, } \\
\text { RCBD } \\
\text { model }\end{array} \\
\begin{array}{c}\text { A posteriori } \\
\text { mean }\end{array}\end{array}$} & \multirow{3}{*}{$\begin{array}{c}\text { Frequentist } \\
\text { analysis, } \\
\text { RCBD } \\
\text { model } \\
\\
\text { i BLUP } \\
\text { mean }\end{array}$} \\
\hline & & \multirow{2}{*}{$\begin{array}{c}A \\
\text { posteriori } \\
\text { mean }\end{array}$} & \multicolumn{2}{|c|}{$\begin{array}{c}95 \% \text { credible } \\
\text { interval }\end{array}$} & \multirow{2}{*}{$\begin{array}{c}\text { A } \\
\text { posteriori } \\
\text { mean- } \\
\text { rank }\end{array}$} & \multicolumn{5}{|c|}{ A posteriori rank at quantile } & & & \\
\hline & & & Lower & Upper & & 0.025 & 0.05 & 0.5 & 0.95 & 0.975 & & & \\
\hline & & t ha $^{-1}$ & $-\mathrm{tr}$ & $\mathrm{a}^{-1}-$ & & & & & & & t ha $^{-1}$ & $\mathrm{tha}^{-1}$ & t ha $^{-1}$ \\
\hline 1 & G14 & 2.34 & 2.05 & 2.61 & 2.7 & 1 & 1 & 2 & 8 & 11 & 2.35 & 2.12 & 2.19 \\
\hline 2 & G19 & 2.26 & 2.00 & 2.52 & 4.4 & 1 & 1 & 3 & 12 & 15 & 2.27 & 2.11 & 2.19 \\
\hline 3 & G18 & 2.19 & 1.93 & 2.45 & 6.7 & 1 & 1 & 5 & 17 & 19 & 2.19 & 2.00 & 2.01 \\
\hline 4 & G5 & 2.16 & 1.92 & 2.41 & 7.7 & 1 & 1.5 & 6.5 & 18 & 20 & 2.16 & 2.12 & 2.20 \\
\hline 5 & G20 & 2.14 & 1.90 & 2.39 & 8.4 & 1 & 2 & 7 & 19 & 21 & 2.14 & 2.06 & 2.10 \\
\hline 6 & G2 & 2.14 & 1.90 & 2.39 & 8.2 & 1 & 2 & 7 & 18 & 21 & 2.14 & 2.03 & 2.05 \\
\hline 7 & G11 & 2.09 & 1.84 & 2.34 & 10.6 & 2 & 2.5 & 10 & 22 & 24 & 2.08 & 1.93 & 1.89 \\
\hline 8 & G17 & 2.09 & 1.85 & 2.34 & 10.6 & 2 & 2 & 10 & 21 & 23 & 2.09 & 2.05 & 2.09 \\
\hline 9 & G21 & 2.08 & 1.84 & 2.33 & 10.9 & 2 & 3 & 10 & 21 & 24 & 2.09 & 2.05 & 2.08 \\
\hline 10 & G23 & 2.06 & 1.82 & 2.31 & 11.9 & 2 & 3 & 11 & 23 & 24.5 & 2.06 & 2.01 & 2.03 \\
\hline 11 & G16 & 2.06 & 1.83 & 2.31 & 11.7 & 2 & 3 & 11 & 22 & 24 & 2.07 & 2.05 & 2.08 \\
\hline 12 & G9 & 2.04 & 1.79 & 2.30 & 12.9 & 2 & 3 & 13 & 23 & 25 & 2.04 & 2.07 & 2.12 \\
\hline 13 & G28 & 2.05 & 1.81 & 2.29 & 12.4 & 2 & 3 & 12 & 23 & 25 & 2.05 & 2.03 & 2.04 \\
\hline 14 & G25 & 2.04 & 1.80 & 2.28 & 13.1 & 3 & 3.5 & 13 & 24 & 25 & 2.03 & 2.05 & 2.08 \\
\hline 15 & G8 & 2.03 & 1.79 & 2.27 & 13.5 & 3 & 4 & 13 & 24 & 26 & 2.02 & 1.94 & 1.90 \\
\hline 16 & G27 & 1.98 & 1.74 & 2.22 & 15.7 & 4 & 5.5 & 16 & 26 & 27 & 1.98 & 1.98 & 1.97 \\
\hline 17 & G26 & 1.97 & 1.72 & 2.21 & 16.4 & 4 & 6 & 17 & 26 & 27 & 1.96 & 1.93 & 1.89 \\
\hline 18 & G24 & 1.95 & 1.71 & 2.19 & 17.3 & 5 & 6.5 & 18 & 26 & 28 & 1.94 & 1.98 & 1.97 \\
\hline 19 & G1 & 1.94 & 1.70 & 2.18 & 17.8 & 5 & 7 & 18 & 27 & 28 & 1.93 & 1.92 & 1.87 \\
\hline 20 & G13 & 1.93 & 1.69 & 2.17 & 18.1 & 5 & 7 & 19 & 27 & 28 & 1.93 & 2.04 & 2.08 \\
\hline 21 & G3 & 1.91 & 1.67 & 2.15 & 19.1 & 6 & 8 & 20 & 27 & 28 & 1.90 & 1.95 & 1.93 \\
\hline 22 & G12 & 1.88 & 1.63 & 2.12 & 20.4 & 7 & 10 & 21 & 28 & 29 & 1.88 & 1.96 & 1.94 \\
\hline 23 & G15 & 1.87 & 1.62 & 2.10 & 21.2 & 8 & 11 & 22 & 28 & 29 & 1.85 & 1.92 & 1.88 \\
\hline 24 & G7 & 1.86 & 1.60 & 2.10 & 21.7 & 9 & 11 & 23 & 29 & 29 & 1.85 & 1.86 & 1.77 \\
\hline 25 & G6 & 1.85 & 1.59 & 2.10 & 21.8 & 9 & 11 & 23 & 29 & 29 & 1.84 & 2.01 & 2.02 \\
\hline 26 & G30 & 1.76 & 1.45 & 2.07 & 24.8 & 10 & 14 & 26 & 30 & 30 & 1.84 & 1.95 & 1.96 \\
\hline 27 & G4 & 1.79 & 1.54 & 2.04 & 24.0 & 12 & 15 & 25 & 30 & 30 & 1.78 & 1.92 & 1.88 \\
\hline 28 & G29 & 1.71 & 1.45 & 1.97 & 26.5 & 17 & 19 & 27.5 & 30 & 30 & 1.69 & 1.85 & 1.76 \\
\hline 29 & G22 & 1.68 & 1.41 & 1.96 & 27.2 & 18 & 20.5 & 28 & 30 & 30 & 1.66 & 1.89 & 1.82 \\
\hline 30 & G10 & 1.68 & 1.40 & 1.95 & 27.3 & 19 & 21 & 28 & 30 & 30 & 1.66 & 1.82 & 1.72 \\
\hline AvSE ${ }^{\S}$ & & 0.128 & & & & & & & & & 0.160 & 0.130 & 0.168 \\
\hline AvSED" & & 0.181 & & & & & & & & & 0.188 & 0.180 & 0.203 \\
\hline
\end{tabular}

${ }^{\dagger}$ Monte Carlo (MC) error in the range 0.0011 to 0.0016 .

‡ BLUP, best linear unbiased predictor.

$\S$ AvSE, average standard error.

" AvSED, average standard error of difference.

of the performance of genotypes in future and from various considerations the assumption of random effects of genotypes becomes more admissible. Using random effect models, the various linear effect parameters were assumed normally distributed with their own variances that were assigned prior distributions. The linear models are as described in standard approaches in textbooks (Cochran and Cox, 1957; Hinkelmann and Kempthorne, 2005). Singh et al. (2010, 2012, 2013) modeled the heterogeneity of error variances in field situations. The error variances are usually assumed constant, while in reality they may vary over the layout. This results in a distribution of indices measuring the heterogeneity of a field or even estimates of broad-sense heritability. In this light, it is more realistic to develop procedures to draw inferences when there is a distribution of error variances and the Bayesian approach is better suited to model such a reality of field experimentation. The analysis of multienvironment trials is another area requiring the strong scope of a Bayesian approach. For example, in the combined analysis of trials, instead of using estimates of generally found heterogeneous error and interaction variances, Edwards and Jannink (2006) 
Table 5. Current a posteriori estimates of standard deviation components for using as priors in analysis of a future trial.

\begin{tabular}{|c|c|c|c|c|}
\hline \multirow[b]{2}{*}{ Parameters $^{\dagger}$} & \multicolumn{2}{|c|}{$\begin{array}{l}\text { A posteriori } \\
\text { distribution }\end{array}$} & \multicolumn{2}{|c|}{$\begin{array}{l}\text { A priori distribution } \\
(\text { Table } 2)^{\ddagger}\end{array}$} \\
\hline & Mean & $\begin{array}{c}\text { Precision } \\
\tau\end{array}$ & Mean & $\begin{array}{c}\text { Precision } \\
\tau\end{array}$ \\
\hline \multicolumn{5}{|c|}{ Incomplete block design } \\
\hline$\sigma_{r}$ & 0.15 & 143.7 & 0 & 20.7 \\
\hline$\sigma_{\mathrm{g}}$ & 0.20 & 557.6 & 0 & 75.4 \\
\hline$\sigma_{e}$ & 0.25 & 1675.5 & 0 & 188.2 \\
\hline$\sigma_{b}$ & 0.28 & 296.2 & 0 & 27.3 \\
\hline \multicolumn{5}{|c|}{ Randomized complete block design } \\
\hline$\sigma_{\mathrm{r}}$ & 0.11 & 302.5 & 0 & 62.4 \\
\hline$\sigma_{g}$ & 0.38 & 846.5 & 0 & 56.4 \\
\hline$\sigma_{e}$ & 0.13 & 206.4 & 0 & 42.6 \\
\hline
\end{tabular}

${ }^{\dagger} \sigma_{r}=$ standard deviation component for replication effects; $\sigma_{g}=$ standard deviation component for genotype effects; $\sigma_{e}=$ standard deviation component for experimental errors; $\sigma_{b}=$ standard deviation component for incomplete blocks within replications effects.

¥ Mean and Precision $(\tau)$ are parameters of $\mathrm{N}(\text { Mean }, \tau)^{+}$, with $\tau=$ variance $^{-1}$ (see Gelman et al. [2004] for notation).

modeled heterogeneity using an exponential of an additive model followed by assigning suitable priors to the variance components of the effects terms of the model.

The choice of priors suited for variance parameters is discussed in Gelman (2006). The inverse $\gamma$ distributions were used as priors for variance parameters but the R2WinBUGS-generated computational errors in several of the fixed values of the hyperparameters in the hierarchy. Further, Gelman (2006) also recommended that $\gamma$ priors not be used for variances. Polson and Scott (2011) recommended that half-Cauchy should replace an inverse- $\gamma$ distribution as a default prior for scale parameters. The performance of the $\gamma$ priors varied over the two designs in the present study and was not the best. With some lower values of $\gamma$ distribution parameters than considered in the present study, WinBUGS could not proceed with the sampling of parameters. The set of priors used in the present study did vary in distribution form and parameters; however, the DIC values were not very different. Thus, in effect, any other priors could be used for a posteriori estimation purposes. However, priors derived from the series of already conducted trials were best to strengthen our belief in the seen SDCs or variance components. This study clearly demonstrates the steps involved in using the data collected from previous trials, which adds a new value to past investment.

In the Bayesian approach, due to its computational nature in every situation, simple or complex, the whole distribution is available in a well-established tool such as R2WinBUGS, and the summary statistics are conveniently available for the parameters of interest including derived ones in terms of model parameters. Such statistics often required are estimates, standard errors, and confidence intervals or quantiles. In many cases, variance components showed substantial differences in their respective means and medians, indicating their skewed distributions. The derived expressions for standard errors under the frequentist approach generally hold good for large samples while we obtain exact values in the Bayesian method, of course with known degree of accuracy depending on the parameters set for convergence. The posterior distributions of the parameters (e.g., SDCs) could be used as priors for analyzing the data that would become available after the next season of the trial. Therefore, these steps provide a successive way of updating the priors with availability of future trial datasets. Since the posteriors use the models describing the plot-wise data, the updated priors based on these posteriors provide a better alternative to priors obtained by fitting the distributions for SDCs, which are based only on the variance component estimates from the trials used for the (current) priors and the current dataset. However, the prior for the trial mean may be evaluated by fitting the distribution to the set of trial means when available.

The rich suites of $\mathrm{R}$-codes facilitate assessing genotypes for their relative performances in terms of ranks and their distributions. While one would like to select the high-ranking genotypes using predicted means, one would also like to see the merit in retaining high ranks in all occurrences or samplings. For the IBD dataset (Table 4), while there were high correlations between the ranks of frequentist and Bayesian approaches, more datasets are needed to evaluate how the selection of high-yielding genotypes is affected.

\section{CONCLUSIONS}

Crop improvement is a continuous process involving regular evaluation of varieties in designed field trials and generating data on responses of the varieties. Thus, valuable information on the varieties' effects and variance components of error and factors with random effects is gathered. Fitting of linear models in crop variety trials evaluated under IBD or RCBD conditions is normally by ANOVA or REML. The frequentist approach is too restrictive in the sense that it is based on the likelihood of the current dataset and uses models where linear parameters and variance parameters are treated as fixed constants and ignores any prior information concerning these parameters. However, the Bayesian approach integrates the prior information available from previous trials with that of the current dataset and provides a more realistic and wider coverage for statistical inference. It also provides an update on the prior information for use in future trials. The illustrative step-by-step procedure presented here is recommended for routine use in statistical analysis of crop genotype evaluations.

\section{APPENDIX}

\section{A.1 Models and Priors for Bayesian Analysis of Data from Incomplete Block Design}

Let $y_{i j k}$ be the response, that is, grain yield, from the $i$ th genotype in the $k$ th incomplete block of the $j$ th replication. It can be modeled as: 


$$
y_{i j k}=\mu+\rho_{j}+\beta_{j k}+g_{i}+\varepsilon_{i j k}
$$

where the parameter $\mu$ is general mean, $\rho_{j}$ is the effect of $j$ th replication, $\beta_{j k}$ is the effect of $k$ th block in $j$ th replication, and $g_{i}$ the effect of $i$ th genotypes. The indices $j=1 \ldots r, k$ $=1 \ldots K$, and $i=1 \ldots v$, where $r$ is number of replications, $v$ is number of genotypes, and $K$ is the number of blocks in each replication. The quantity $\varepsilon_{i j k}$ is random error for the plot under $i$ th genotype, $k$ th block of the $j$ th replication. The block effects $\beta_{j k}$ and $\varepsilon_{i j k}$ are assumed independent and normally distributed with means zero and variances $\sigma_{\beta}^{2}$ and $\sigma_{\varepsilon}^{2}$, respectively. In the classical analysis model the unknown parameters, $\mu, \rho_{j}(j=1 \ldots r), g_{i}(i=1 \ldots \nu), \sigma_{\beta}^{2}$, and $\sigma_{\varepsilon}^{2}$ are assumed fixed and are estimated using either least squares method for fixed effects of a normal linear model or maximum likelihood method for all parameters (see Cochran and Cox, 1957; Hinkelmann and Kempthorne, 2005). Using matrix notation, where ' $\sim$ ' under a symbol denotes a matrix and an underscore a vector, the model (1) can be written as:

$$
\underline{\mathbf{y}}=\mu \underline{\mathbf{J}}+\underline{\mathbf{D}}_{1} \underline{\rho}+{\underset{\sim}{2}}_{2} \underline{\beta}+\underline{\sim}_{\underline{3}} \underline{\gamma}+\underline{\varepsilon}
$$

where the vector $\underline{\mathbf{y}}$ is vector of observations $\left(\gamma_{i j k}\right)$; vectors $\rho, \beta$, and $\gamma$ are vectors of replication effects, block effects within replications, and genotype effects, respectively; vector $\underline{\mathbf{J}}$ is a vector of all $1 \mathrm{~s}$; and matrices $\mathbf{D}_{1}, \mathbf{D}_{2}$, and $\mathbf{D}_{3}$ are incidence matrices of replication, block, and genotype effects, respectively. For estimation of heritability and genetic gain due to selection, we assume that vector $\gamma$ has a multivariate normal distribution, $\operatorname{MN}\left(\underline{\mathbf{0}}, \sigma_{\mathrm{g} \sim}^{2} \mathbf{I}\right)$, where $\underset{\sim}{\mathbf{I}}$ is an identity matrix of appropriate order, in this case of order $v$. We further assume that the vector of replication effects, $\underline{\rho}$, follows $\operatorname{MN}\left(\underline{\mathbf{0}}, \sigma_{\mathrm{r}}^{2} \mathbf{I}\right)$. All sets of random variables used here are assumed independent. The variance-covariance matrix of $\mathbf{y}$ in (2) is $\underset{\sim}{\mathbf{V}}=V(\underline{\mathbf{y}})=\underline{\sim}_{1} \mathbf{D}_{\sim}^{\prime} \sigma_{\mathrm{r}}^{2}+\mathbf{D}_{2} \mathbf{D}_{\sim}^{\prime} \sigma_{\beta}^{2}+\underline{\sim}_{3} \mathbf{D}_{3}^{\prime} \sigma_{\mathrm{g}}^{2}+\sigma_{\varepsilon}^{2} \mathbf{I}$. The likelihood function of $\mu, \sigma_{\mathrm{r}}^{2}, \sigma_{\beta}^{2}, \sigma_{\mathrm{g}}^{2}$, and $\sigma_{\varepsilon}^{2}$ in terms of the observations is:

$$
\begin{aligned}
& p\left(\underline{\mathbf{y}} \mid \mu, \sigma_{\mathrm{r}}^{2}, \sigma_{\beta}^{2}, \sigma_{\mathrm{g}}^{2}, \sigma_{\varepsilon}^{2}\right)=N\left(\underline{\mathbf{y}} \mid \mu,{\underset{\sim}{1}}_{\sim}^{\mathbf{D}_{1}^{\prime}} \sigma_{\mathrm{r}}^{2}\right. \\
& \left.+\mathbf{D}_{2} \underset{\sim}{\mathbf{D}_{2}^{\prime}} \sigma_{\beta}^{2}+\mathbf{D}_{3} \mathbf{D}_{3}^{\prime} \sigma_{\mathrm{g}}^{2}+\sigma_{\varepsilon}^{2} \mathbf{I}\right)
\end{aligned}
$$

where $M N(\underline{\mathbf{x}} \mid \underline{\mathbf{a}}, \underset{\sim}{\mathbf{b}})$ stands for the probability density function of the normal random vector with mean vector $\underline{\mathbf{a}}$ and variance covariance matrix $\underset{\sim}{\mathbf{b}}$ and evaluated at point vector $\underline{\mathbf{x}}$. The expression of this density is available in standard texts (Gelman et al., 2004).

\section{A.2 Heritability and Genetic Advance Due to Selection}

Expressing also the estimates of genotypic variance by $\sigma_{\mathrm{g}}^{2}$ and experimental error (environmental) variance by $\sigma_{\mathrm{e}}^{2}$, the broad-sense heritability on a mean-basis is given by $h^{2}=\sigma_{\mathrm{g}}^{2} /\left(\sigma_{\mathrm{g}}^{2}+\sigma_{\mathrm{e}}^{2} / r\right)$, where $r$ is the number of replications. Another expression for heritability was proposed by Cullis et al. (2006), which corrects for the bias better than $h^{2}$ (Piepho and Möhring, 2007), is $h_{\mathrm{C}}^{2}=1-\bar{\nu}_{\mathrm{BLUP}} /\left(2 \sigma_{\mathrm{g}}^{2}\right)$, where $\bar{\nu}_{\mathrm{BLUP}}$ is the average variance of pair-wise differences between the best linear unbiased predictors (BLUPs). Thus for a chosen heritability, the GA based on mean over replications and for a selection intensity of $p$ is $C \sigma_{\mathrm{g}} h^{*} / \bar{Y}$, where $C$ is a constant given by $C=\frac{1}{p \sqrt{2 \pi}} e^{-z_{p}^{2} / 2}$ and $z_{p}$ is the upper $p$ quantile of standard normal distribution, $h h^{\star}$ is the square root of the heritability, $h^{2}$ or $h_{\mathrm{C}}^{2}$, and $\bar{Y}$ is mean response (Kempthorne, 1983; Singh et al., 2012). The quantity $C$ is 1.4 for $20 \%$ intensity of selection. We also obtained the realized gain without using any heritability expression (Piepho and Möhring, 2007). While the standard error of heritability, $h^{2}$, and associated genetic gain was obtained from Win2BUGS standard output, the standard errors of $h_{\mathrm{C}}^{2}$ and associated gain were obtained using bootstrap procedure in the R-package on the simulated values of BLUPs as the $h_{\mathrm{C}}^{2}$ expression being complicated could not be included in the WinBUGS model file.

\section{A.3 Priors}

In this study, we pursue the Bayesian approach where all parameters are assumed to follow some joint marginal or independent statistical distributions known as priors. Gelman (2006) reviewed a number of options for noninformative priors for scale parameters in hierarchical models, and his recommendation included the use of uniform, half- $t$, half-Chauchy, and half-normal families of distributions. Crossa et al. (2010) used an inverse- $\gamma$ distribution as a prior for variance components; however, Gelman (2006) suggested not using an inverse- $\gamma$ family as priors due to the resulting sensitivity of results. To choose the best priors among a set of candidate priors, criteria given in the following have been presented in standard texts such as Gelman et al. (2004) and Carlin and Louis (2009).

There might be multiple sources of prior information including classes of priors recommended for variance or SDCs. A question naturally arises as to which would be the best priors among the set of priors considered.

A number of a priori distributions have been used for the variance components:

1. $\mathrm{P}_{0}$ : the priors obtained from analysis of series of 20 datasets (Table 1). The yield values and standard deviation (or the variance) components were positive, so their distributions were constrained to the positive values of normal distributions. Thus, for $\sigma_{r}$, the distribution can be denoted by $\sigma_{\mathrm{r}} \sim N\left(0, \sigma^{2}=0.048356\right)^{+}$. In a Bayesian context, a precision parameter $\tau$ is used, where $\tau=\sigma^{-2}$ (inverse of variance), instead of variance 
of normal distribution. This prior, using the WinBUGS code, is expressed as $\sigma_{\mathrm{r}} \sim \operatorname{dnorm}(0, \tau)^{*} I(0$,$) ,$ where $I(0$,$) restricts the generated values of \sigma_{\mathrm{r}} \sim$ $N\left(0, \sigma^{2}\right)$ in the positive range. Similarly the priors for other parameters were taken as positive parts of normal distributions: $\sigma_{\mathrm{b}} \sim N\left(0, \tau^{-1}=0.036634\right)^{+}, \sigma_{\mathrm{g}} \sim$ $N\left(0, \tau^{-1}=0.013271\right)^{+}, \sigma_{\mathrm{e}} \sim N\left(0, \tau^{-1}=0.005314\right)^{+}$, and $\mu \sim N\left(2.26, \tau^{-1}=0.128522\right)^{+}$.

2. $\mathrm{P}_{1}$ : the priors for the standard deviation components $\sigma_{\mathrm{e}}, \sigma_{\mathrm{g}}, \sigma_{\mathrm{r}}$, and $\sigma_{\mathrm{b}}$ follow uniform $(0, \theta=50)$.

3. $\mathrm{P}_{2}$ : the priors for the standard deviation components $\sigma_{\mathrm{e}}, \sigma_{\mathrm{g}}, \sigma_{\mathrm{r}}$, and $\sigma_{\mathrm{b}}$ follow a normal distribution $N\left(0, \tau^{-1}\right.$ $=10,000)^{\star} I(0$,$) using notation of Gelman (2006).$

4. $\mathrm{P}_{3}$ : the priors for the standard deviation components $\sigma_{\mathrm{e}}, \sigma_{\mathrm{g}}, \sigma_{\mathrm{r}}$, and $\sigma_{\mathrm{b}}$ follow the positive part of the $t$-distribution and denoted as $t(0, c, v)^{+}$or equivalently as $d t(0, c, v) I(0$,$) . Here, c$ is noncentrality parameter and $v$ is the degree of freedom of the $t$-distribution. The values of $c$ and $v$ are set at 5 and 2, respectively.

5. $\mathrm{P}_{4}$ : the priors of $\mathrm{P}_{3}$ with $c=2$ and $v=4$.

6. $\mathrm{P}_{5}$ : the priors for precision parameters (i.e., inverse of the variances, $\sigma_{\mathrm{e}}, \sigma_{\mathrm{g}}, \sigma_{\mathrm{r}}$, and $\sigma_{\mathrm{b}}$ ) follow a $\gamma$ distribution $(0.5,0.5)$.

The sets of a priori distributions $\mathrm{P}_{0}$ through $\mathrm{P}_{5}$ were used for analysis of IBD data. For analysis of RCBD data, a priori distributions would be needed only for $\mu$, $\sigma_{\mathrm{e}}, \sigma_{\mathrm{g}}$, and $\sigma_{\mathrm{r}}$, (Table 1,2). The a priori distribution sets for $\mu, \sigma_{\mathrm{e}}, \sigma_{\mathrm{g}}$, and $\sigma_{\mathrm{r}}$ will be denoted by $\mathrm{Q}_{0}$ through $\mathrm{Q}_{5}$ corresponding to $\mathrm{P}_{0}$ through $\mathrm{P}_{5}$ distributions.

\section{A.4 A Posteriori Distribution}

To express various terminologies in the Bayesian context, standard texts may be consulted (Gelman et al., 2004; Carlin and Louis, 2009). The joint prior, based on individual priors, will be assumed as:

$$
\begin{aligned}
& p\left(\mu, \underline{\rho}, \underline{\beta}, \underline{\gamma}, \sigma_{\mathrm{r}}, \sigma_{\beta}, \sigma_{\mathrm{g}}, \sigma_{\varepsilon}\right) \propto N\left(\underline{\rho} \mid \underline{\mathbf{0}}, \sigma_{\mathrm{r}}^{2}\right) f_{\mathrm{r}}\left(\sigma_{r} \mid \underline{\theta}_{0 \mathrm{r}}\right) \\
& N\left(\underline{\beta} \mid \underline{\mathbf{0}}, \sigma_{\beta}^{2}\right) f_{\beta}\left(\sigma_{\beta} \mid \underline{\theta}_{0 \beta}\right) \bullet \\
& N\left(\underline{\gamma} \mid \underline{\mathbf{0}}, \sigma_{\mathrm{g}}^{2}\right) f_{\mathrm{g}}\left(\sigma_{\mathrm{g}} \mid \underline{\theta}_{0 \mathrm{~g}}\right) f_{\mathrm{e}}\left(\sigma_{\mathrm{e}} \mid \underline{\theta}_{0 \mathrm{e}}\right)
\end{aligned}
$$

where $f_{x}\left(\sigma_{x} \mid \underline{\theta}_{0 x}\right)$ stands for the probability density function of hyperparameters assumed as random variable denoted by $\sigma_{x}$ and evaluated at $\sigma_{x}$ where $\underline{\theta}_{0 x}$ is the associated scalar or vector of the parameters of known value. The joint a posteriori density of $\mu, \underline{\boldsymbol{\rho}}, \underline{\boldsymbol{\beta}}, \underline{\gamma}, \sigma_{\mathrm{r}}, \sigma_{\beta}, \sigma_{\mathrm{g}}$, and $\sigma_{\varepsilon}$ can be written, given $\gamma$, as:

$$
\begin{aligned}
& p\left(\mu, \underline{\rho}, \underline{\beta}, \underline{\gamma}, \sigma_{\mathrm{r}}, \sigma_{\beta}, \sigma_{\mathrm{g}}, \sigma_{\varepsilon} \mid \underline{\mathbf{y}}\right) \propto N\left(\gamma \mid \mu, \underline{\mathbf{D}}_{\sim} \mathbf{D}_{1}^{\prime} \sigma_{\mathrm{r}}^{2}+\underline{\mathbf{D}}_{2} \mathbf{D}_{2}^{\prime} \sigma_{\beta}^{2}\right. \\
& \left.+\mathbf{D}_{3} \mathbf{D}_{3}^{\prime} \sigma_{\mathrm{g}}^{2}+\sigma_{\varepsilon \sim}^{2} \mathbf{I}\right) \cdot \\
& N\left(\underline{\rho} \mid \underline{\mathbf{0}}, \sigma_{\mathrm{r}}^{2}\right) f_{\mathrm{r}}\left(\sigma_{\mathrm{r}} \mid \underline{\theta}_{0 \mathrm{r}}\right) N\left(\underline{\beta} \mid \underline{\mathbf{0}}, \sigma_{\beta}^{2}\right) f_{\beta}\left(\sigma_{\beta} \mid \underline{\theta}_{\beta \beta}\right) \cdot \\
& N\left(\underline{\gamma} \mid \underline{\mathbf{0}}, \sigma_{\mathrm{g}}^{2}\right) f_{\mathrm{g}}\left(\sigma_{\mathrm{g}} \mid \underline{\theta}_{\mathrm{g}}\right) f_{\mathrm{e}}\left(\sigma_{\mathrm{e}} \mid \underline{\theta}_{\theta_{e}}\right)
\end{aligned}
$$

For RCBD datasets, the above expressions can be modified by dropping the terms corresponding to incomplete blocks within replications.

\section{A.5 Computations using R2WinBUGS}

The R2WinBUGS software (Spiegelhalter et al., 2003) uses a text file containing the data model with parameters, and information on the parameters' a priori distributions. It can also include derived parameters, such as, contrasts for comparisons of genotypic effects, genotypic variance components, heritability and genetic gain due to selection. Based on the derived a posteriori density of the parameters, R2WinBUGS provides various statistics: the goodness-of-fit or discrepancy statistics, deviance information criterion (DIC), effective number of parameters $\left(p_{\mathrm{D}}\right)$, and the expected values (a posteriori estimates) of the parameters of interest, Monte Carlo (MC) error, and quantiles of their posterior distribution. The DIC, the smaller the better, was used to choose the prior. In the BUGS function, the iterations were set at 1 million, simulations at 10,000 and chains at three. These served the purpose of convergence. The R-codes ( $\mathrm{R}$ Development Core Team, 2009) were used to carry out calculations of standard error of differences and the posterior average and quantiles of ranks distribution. For the IBD and RCBD datasets, the associated codes are given in the following.

\section{A.6 Bayesian model under R2WinBUGS}

The discrepancy statistics DIC and effective number of parameters $p_{\mathrm{D}}$ are computed. The DIC, the smaller the better, was used to choose the prior.

The posterior distribution of the quantities of interest, including heritability, experimental error coefficient of variation and genetic gain, were obtained using the R2WinBUGS and R-package and the summary in terms of mean and confidence intervals. Selection of genotypes was based on mean predicted values, and the average rank of the genotypes. The models using WinBUGS notations and $\mathrm{R}$-codes for an example dataset are given in the following.

\section{A.7 IBD Model File ('Bug' Extension) Contents \\ \#\#\#\#\#\#\#\#\#\#\#\#\#\#\#\# IBD data analysis \\ model \{ \\ for (i in 1:N)\{ \\ $y[i] \sim \operatorname{dnorm}(\mathrm{mu}[\mathrm{i}]$, tau.e) \\ $m u[i]<-m+\operatorname{rho[rp[i]]}+\operatorname{bet}[\mathrm{rp}[i], b \mid[i]]+g[\operatorname{gn}[i]]$ \\ \} \\ \# rho[1...NR-1] Rep effects result into rho[NR] \\ for (i in 1:(NR -1)) \{rho[i] dnorm(0, tau. $r)\}$ \\ rho[NR] <- - sum(rho[1:(NR-1)]) \\ for (i in 1:NR) \{ for(j in 1: (NB-1)) \{ bet[i,j] dnorm(0,tau.b) \} \# except \\ the last, block effects within repl. \\ bet[i,NB] <- -sum(bet[i,1:(NB-1)]) \# last block in the replication \\ \}}


\# g[1....NG-1] Geno effects result into g[NG]

for (i in 1: (NG-1)) $\{\mathrm{g}[\mathrm{i}] \sim \operatorname{dnorm}(0$, tau.g) $\}$

$g[N G]<--\operatorname{sum}(g[1:(N G-1)])$

\#...........priors here

$\mathrm{m} \sim \operatorname{dnorm}(2.26,7.78) \mid(0$,

sig.r dnorm $(0,20.67)(0$,

sig.b dnorm(0, 27.29)!(0,)

sig.g dnorm $(0,75.35)(0$,

sig.e dnorm $(0,188.17)(0$,

\#-_-- Parameters of interest

tau.e $<-1 /$ (sig.e ${ }^{*}$ sig.e)

tau.b $<-1 /($ sig.b* sig.b)

tau.g $<-1 /\left(\right.$ sig.g ${ }^{*}$ sig.g)

tau. $r<-1 /\left(\right.$ sig..$^{*}$ sig. $\left.r\right)$

sig2 $\mathrm{g}<-$ (sig.g*sig.g)

sig2e $<-\left(\right.$ sig. $e^{*}$ sig.e $)$

sig2b $<-($ sig.b*sig.b)

sig2 $r<-\left(\right.$ sig. $r^{*}$ sig. $\left.r\right)$

\# Prediction of parameters of interest-means, heritability, SEs

for (i in 1: NG) $\{$ PredG[i]<- $m+g[i]\}$

\# this heritability is on mean-basis

h2<- tau.e/(tau.e + tau.g/NR)

\# Genetic advance (GA) due to 20\% selection \& 10\%, 5\% selection

$\mathrm{K}=1.4,1.755,2.063$

GA20 <- 100*1.4/mn/sqrt(tau.g ${ }^{*}(1+$ tau.g/NR/tau.e))

GA10 <- 100*1.755/mn/sqrt(tau.g*(1+tau.g/NR/tau.e))

GA5 <- 100*2.063/mn/sqrt(tau.g*(1+tau.g/NR/tau.e))

\# Simulate realized gain simGA20

\# NG $=30 ; 20 \%$ (of 30) $=6$

\# NLow20<- NG*(1-0.20)

for(i in 1:NLow20)\{low20[i]<- ranked(PredG[],i)\}

simGA20<- $100 *(\mathrm{NG} /(\mathrm{NG}-\mathrm{NLow} 20) \quad-1)^{*}(1-\quad$ mean(low20[])/ mean(PredG[]))

\# Simulate realized gain simGA10

\# NG $=30 ; 10 \%$ (of 30) $=3$

\# NLow10<- NG*(1-0.10)

for(i in 1:NLow10)\{low10[i]<- ranked(PredG[],i)\}

simGA10<- $100^{*}(\mathrm{NG} /(\mathrm{NG}-\mathrm{NLow10}) \quad-1)^{*}(1-\quad$ mean(low10[])/ mean(PredG[]))

\# Simulate realized gain simGA5

\# NG $=30 ; 5 \%$ (of 30) $=2$

\# NLow5<- NG*(1-0.05)

for(i in 1:NLow5)\{low5[i]<- ranked(PredG[],i)\}

simGA5<- $\quad 100 *(N G /(N G-N L o w 5) \quad-1)^{*}(1-\quad$ mean $(l o w 5[]) /$ mean(PredG[]))

\# CV\%

CVpc $<-100 /$ sqrt(tau.e)/mn

\}

\# end of BUGS codes

For the RCB dataset, the top lines can be replaced, following the comment but without any incomplete block effects parameters 'bet[]' as follows: for $(\mathrm{i}$ in $1: \mathrm{N})\{$

$y[i] \sim \operatorname{dnorm}(m u[i]$, tau.e)

$m u[i]<-m+$ rho[rp[i]] + g[gn[i]]

\}

The lines or text sections involving parameters such as tau.g, sig.b, and sig2b should be removed.

\section{A.8 Model terms used for the other priors}

1. Uniform distributions

sig.r $\sim \operatorname{dunif}(0,50)$

sig.b dunif $(0,50)$

sig.g $\sim \operatorname{dunif}(0,50)$

sig.e $\sim \operatorname{dunif}(0,50)$

2. Half t-distributions

sig. $r \sim \mathrm{dt}(0,5,2)(0$,

sig.b dt $(0,5,2)(0$,

sig.g $\sim \mathrm{dt}(0,5,2)((0)$,

sig.e $\sim \operatorname{dt}(0,5,2)(0$,

3. Gamma distribution

tau.r dgamma $(.5,5)$

tau.b dgamma(.5,.5)

tau.g dgamma(.5,.5)

tau.e $\sim$ dgamma $(.5, .5)$

\section{A.9 R-Codes for Dataset on IBD}

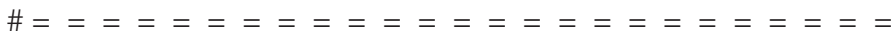
\#load packs

library(lattice)

library(coda)

library(R2WinBUGS)

\# Read IBD (incomplete block design) data with columns of Replications [Rep], Blocks within replications [Blk], Genotypes[Geno] and responses [Yld] \#

Data<- read.table("ExampleIBDData.txt", header = TRUE) y<- Data\$Yld

$\mathrm{rp}<-$ Data\$Repgn<- Data\$Geno

bl<- Data\$Blk

\# The number of replications, genotypes, total number of observtion. These can be computed using suitable functions also.

$\mathrm{NR}<-3$

$\mathrm{NB}<-6$

$N G<-30$

$\mathrm{N}<-\mathrm{NR} R^{*} \mathrm{NG}$

$N B R 1<-N R^{*}(N B-1)$ 
$\operatorname{print}(\mathrm{cbind}(\mathrm{NR}, \mathrm{NB}, \mathrm{NG}, \mathrm{N}))$

$\mathrm{mn}<-$ mean $(\mathrm{y})$

$\mathrm{mn}$

\# Set the numbers of genotypes below top lines at the chosen selection intensity

NLow20<- $\mathrm{NG}^{*}(1-0.20)$ \#at 20\% intensity

NLow $10<-\mathrm{NG}^{*}(1-0.10)$ \#at 10\% intensity

NLow5<- 28 \#NG*(1-0.05) \#at 5\% intensity

print(cbind(NLow20, NLow10, NLow5))

$\#$ \#.......data

data<- list(“mn”, "y”,"rp”,"gn","bl”, “NR”,"NB”,"NG”, “N”, “NLow20”, "NLow10", "NLOw5")

data

\#.......initial values

inits $1<-\operatorname{list}(m=2$, rho $=c($ rep $(.01, N R))$, bet $=c($ rep $(.02, N B R 1)), g=$ $c($ rep $(.01, N G))$, sig.e $=1$, sig.r $=1.0$, sig. $b=.53$, sig.g $=1.22)$

inits2<- list $(m=2$, rho $=c(\operatorname{rep}(.1, N R))$, bet = c(rep(.01, NBR1)), $g=$ $c($ rep $(.02, N G))$, sig.e $=1.1$, sig.r $=1.15$, sig.b $=.68$, sig.g $=1.422)$ inits3 $<-$ list $(m=2$, rho $=c($ rep $(.05, N R))$, bet $=c($ rep(.02, NBR1)), $g$ $=c(r e p(.01, N G))$, sig.e $=1.05$, sig.r $=1.25$, sig.b $=1.35$, sig.g $=$ 1.522)

inits <- list(inits1, inits2, inits3)

\#.....parameters

parameters <- c(“m”,"sig.r”,"sig.g”,"sig.e”, "sig.b”, "sig2 g”, "sig2e","sig2b","sig2r", "PredG”, "h2”, "GA20", "GA10”,

"GA5","simGA20”,"simGA10", "simGA5","CVpc”)

\#.....run BUGS

ibd.sim <- bugs(data, inits, parameters, "IBD.bug", n.chains = 3,

n.iter $=1000000$, n.sims $=10000$, debug $=$ TRUE)

\# Use the simulated values for developing inferential summaries attach.bugs(ibd.sim)

\# bootstrap heritability (h2C) and genetic advance (herit[1:2])

library(boot)

\# function to obtain h2C

herit $<-$ function(data, indices, $\mathrm{mn}=\mathrm{mn})\{$

$\mathrm{d}<-$ data[indices,]

\#............Calc Sigma2G

Sig2G<- mean(apply(d[,1:NG],1, var))

\#.............Calc var-cov and avSED

$\operatorname{cov}<-\operatorname{cov}(\mathrm{d}[$ indices, $1: \mathrm{NG}])$

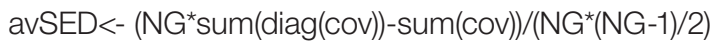

avSED<- sqrt(avSED)

h2C1<- 1- avSED^2/2/Sig2G

Gain20<- 100*1.4*sqrt(Sig2G*h2C1)/mn

herit<- c(h2C1, Gain20)

return(herit)

\}

\# bootstrapping with 1000 replications

NBoots $<-1000$

Results $<-$ boot(data $=$ PredG[,], statistic $=$ herit, $R=$ NBoots, $m n$ $=\mathrm{mn})$

results $\$$ t0

$\operatorname{par}(\mathrm{mfrow}=\mathrm{c}(2,2))$

plot(results, index = 1) \# h2

plot(results, index = 2) \# gain

$H G<-\operatorname{array}(0, \operatorname{dim}=\mathrm{c}($ NBoots,2))

$H G<-$ results $\$ \mathrm{t}$

print(quantile(HG[,1], c(0.025, 0.05,0.5, 0.95, 0.975)))
$\operatorname{print}(\operatorname{cbind}(\operatorname{mean}(\mathrm{HG}[, 1])$, sd(HG[,1])))

print(quantile(HG[,2], c(0.025, 0.05,0.5, 0.95, 0.975)))

print(cbind(mean(HG[,2]), sd(HG[,2])))

\# get 95\%,90\% confidence intervals

boot.ci(results, type = "perc", conf = 0.95, index =1) \# h2C

boot.ci(results, type = "perc", conf = 0.90, index =1) \# h2C

\# get 95\%,90\% confidence intervals

boot.ci(results, type = "perc", conf = 0.95, index = 2) \# GA20\%

boot.ci(results, type = "perc", conf $=0.90$, index =2) \# GA20\%

\#........

\# Compute ranks etc...

\# Get predicted means: mnPred of length NG

$\mathrm{mnPred}<-\operatorname{array}(0, \operatorname{dim}=\mathrm{NG})$

for(j in 1:NG) mnPred[j]<- mean(PredG[,j])

\#.......... RNK: Find rank (and assign Highest = 1). Thus, $\mathrm{RNK}()=$ $\mathrm{n}+1-\operatorname{rank}()$

\# R-core gives: rank $(x)$ asssigns 1 to the lowest value of $x$.

RNK<- function(A,i) \{

\# rank of i-th (entries in i-th position) in A

rnkVec $<-$ length $(A)+1-\operatorname{rank}(A)$

rnkVec[i] $\}$

mean(apply(PredG[,1:NG],1, function(A,i) RNK(A, i), i = 5))

\#Means of ranks of the entries: Max values $>>>$ rank 1

$\operatorname{MnRnk}<-\operatorname{array}(0, \operatorname{dim}=\mathrm{NG})$

for(j in 1:NG) MnRnk[j]<- mean(apply(PredG[,1:NG],1, function(A,i)

$\operatorname{RNK}(A, i), i=j))$

\# quantile of ranks of entries

Ranks<- $\operatorname{array}(0, \operatorname{dim}=\mathrm{c}(\mathrm{NG}, 5))$ \#here: 5 is length(c(0.025, $0.05,0.5,0.95,0.975))$

\# distribution of ranks of each entries

for(j in $1: N G)\{$

Ranks[j,]<- quantile(apply(PredG[,1:NG],1, function(A,i) RNK(A,i), i = j), $c(0.025,0.05,0.5,0.95,0.975))$

\}

print(cbind(1:NG,mnPred,NG+1-rank(mnPred), MnRnk, $\operatorname{rank}(\mathrm{MnRnk})$, Ranks))

For the RCBD dataset, the incomplete block term and associated terms can be removed (bet[], tau.b, sig.b, sig2b, $\mathrm{NB}$ ). These are also available from the authors on request.

\section{Acknowledgments}

The authors thankfully acknowledge contributions of anonymous reviewers for suggestions to include priors extracted from a series of conducted trials and another estimator of heritability and several other suggestions; Professor Y.P. Chaubey, Concordia University, Montreal, a reviewer, and the Associate Editor for valuable discussions on updating the priors; and an editor from Scriptoria for improving the language of the presentation.

\section{References}

Carlin, B.P., and T.A. Louis. 2009. Bayesian methods for data analysis. Chapman and Hall, Boca Raton, FL.

Cochran, W.G., and G.M. Cox. 1957. Experimental design. John Wiley \& Sons, New York, NY.

Coombes, N. 2009. DiGGer design search tool in R. http://www. austatgen.org/software/ (accessed 15 July 2014).

Crossa, J., G. de los Campos, P. Pérez, D. Gianola, J. Burgueño, J.L. 
Araus, D. Makumbi, R.P. Singh, S. Dreisigacker, J. Yan, V. Arief, M. Banziger, and H.J. Braun. 2010. Prediction of genetic values of quantitative traits in plant breeding using pedigree and molecular markers. Genetics 186:713-724. doi:10.1534/ genetics. 110.118521

Cullis, B.R., A. Smith, and N. Coombes. 2006. On the design of early generation variety trials with correlated data. J. Agric. Biol. Environ. Stat. 11:381-393. doi:10.1198/108571106X154443

de los Campos, G., H. Naya, D. Gianola, J. Crossa, A. Legarra, E. Manfredi, K. Weigel, and J.M. Cotes. 2009. Predicting quantitative traits with regression models for dense molecular markers and pedigree. Genetics 182:375-385. doi:10.1534/genetics.109.101501

Edwards, J.W., and J.L. Jannink. 2006. Bayesian modeling of heterogeneous error and genotype $\times$ environment interaction variances. Crop Sci. 46:820-833. doi:10.2135/cropsci2005.0164

Federer, W.T. 1955. Experimental design: Theory and application. Macmillan, New York. Reprinted by the Oxford and IBH Publishing Co. 1967, 1974.

Fisher, R.A. 1990. Statistical methods, experimental design, and scientific inference: A re-issue of statistical methods for research workers, the design of experiments, and statistical methods and scientific inference. Oxford Univ. Press, Oxford, UK.

Forkman, J., and H-P. Piepho. 2012. Performance of empirical BLUP and Bayesian prediction in small randomized complete block experiments. J. Agric. Sci., Cambridge 151:381-395.

Gelman, A., J.B. Carlin, H.S. Stern, and D.B. Rubin. 2004. Bayesian data analysis. 2nd ed. Chapman and Hall, Boca Raton, FL.

Gelman, A. 2006. Prior distributions for variance parameters in hierarchical models. Bayesian Anal. 1:515-533. doi:10.1214/06-BA117A

Hallander, J., P. Waldmann, C. Wang, and M.J. Sillanpää. 2010. Bayesian inference of genetic parameters based on conditional decompositions of multivariate normal distributions. Genetics 185:645-654. doi:10.1534/genetics.110.114249

Hinkelmann, K. 1975. Design of genetical experiments. In: J.N. Srivastva, editor, A survey of statistical design and linear models. North Holland, Amsterdam. p. 243-269.

Hinkelmann, K., and O. Kempthorne. 2005. Design and analysis of experiments, Vol. 2: Advanced experimental design, Wiley Interscience, New York.

John, J.A., and E.R. Williams. 1995. Cyclic and computer generated designs. 2nd ed. Chapman and Hall, London.

Kempthorne, O. 1983. The design and analysis of experiments. R.E. Krieger Publishing Co., Malabar, FL.

Leonard, T. 1975. A Bayesian approach to the linear model with unknown equal variances. Technometrics 17:95-102. doi:10.10 80/00401706.1975.10489277

Lindley, D.V. 1971. The estimation of many parameters. In: V.P., Godambe, and D.A. Sprott, editors, Foundations of statistical inference. Holt, Rinehart and Winston, Toronto. p. 435-455.

Mathew, B., A.M. Bauer, P. Koistinen, T.C. Reetz, J. Léon, and M.J. Sillanpää. 2012. Bayesian adaptive Markov chain Monte Carlo estimation of genetic parameters. Heredity 109:235-245. doi:10.1038/hdy.2012.35
Parsad, R., and V.K. Gupta. 2007. Design resources server. Indian Agricultural Statistics Research Institute (ICAR), New Delhi 110 012, India. http://www.iasri.res.in/design/ (accessed 8 July 2014).

Patterson, H.D., and E.R. Williams. 1976. A new class of resolvable incomplete block designs. Biometrika 63:83-92. doi:10.1093/ biomet/63.1.83

R.W., Payne, editor. 2013. The guide to GenStat release 16. Part 2: Statistics. VSN International, Hemel Hempstead, UK.

Piepho, H.P., and J. Möhring. 2007. Computing heritability and selection response from unbalanced plant breeding trials. Genetics 177:1881-1888. doi:10.1534/genetics.107.074229

Polson, N.G., and J.G. Scott. 2011. On the half-Cauchy prior for a global scale parameter. Cornell University Library. http://arxiv. org/abs/1104.4937 (accessed 21 July 2014).

R Development Core Team. 2009. R: A language and environment for statistical computing. R Foundation for Statistical Computing, Vienna, Austria, ISBN 3-900051-07-0.

SAS Institute. 2011. The SAS system for Windows. Release 9.2. SAS Inst., Cary, NC.

Singh, M., and S. Ceccarelli. 1995. Estimation of heritability using variety trials data from incomplete blocks. Theor. Appl. Genet. 90:142-145.

Singh, M., Y.P. Chaubey, A. Sarker, and D. Sen. 2010. Modelling unstructured heterogeneity along with spatially correlated errors in field trials. J. Ind. Soc. Agric. Stat. 64:313-321.

Singh, M., M. van Ginkel, A. Sarker, R.S. Malhotra, M. Imtiaz, and S. Kumar. 2012. Increasing precision of even otherwise wellrun trials by capturing heterogeneity of plot error variances. Agric. Res. 1:285-294. doi:10.1007/s40003-012-0024-8

Singh, M., W. Tadesse, A. Sarker, F. Maalouf, M. Imtiaz, F. Capettini, and M. Nachit. 2013. Capturing the heterogeneity of the error variances of a group of genotypes in crop cultivar trials. Crop Sci. 53:811-818. doi:10.2135/cropsci2012.11.0637

Smith, A.B., B.R. Cullis, and R. Thomson. 2005. The analysis of crop cultivar breeding and evaluation trials: An overview of current mixed model approaches. J. Agric. Sci. 143:449-462. doi:10.1017/S0021859605005587

Spiegelhalter, D., A. Thomas, N. Best, and D. Lunn. 2003. WinBUGS Version 1.4 user manual. Medical Research Council Biostatistics Unit, Cambridge, UK.

Theobald, C.M., M. Talbot, and F. Nabugoomu. 2002. A Bayesian approach to regional and local-area prediction from crop variety trials. J. Agric. Biol. Environ. Stat. 7:403-419. doi:10.1198/108571102230

Theobald, C.M., and M. Talbot. 2002. The Bayesian choice of crop variety and fertilizer dose. Appl. Stat. 51:23-36.

Whitaker, D., E.R. Williams, and J.A. John. 2009. CycDesigN 4.0: A package for the computer generation of experimental designs. CycSoftware Ltd., Naseby, New Zealand.

Williams, E., H.P. Piepho, and D. Whitaker. 2011. Augmented p-rep designs. Biom. J. 53:19-27. doi:10.1002/bimj.201000102 